\title{
Price of pain: population-based cohort burden of disease analysis of medication cost of herpes zoster and postherpetic neuralgia
}

This article was published in the following Dove Press journal: Journal of Pain Research

3 August 2016

Number of times this article has been viewed

\author{
Kevin J Friesen' \\ Jamie Falk' \\ Silvia Alessi-Severini' \\ Dan Chateau ${ }^{2}$ \\ Shawn Bugden' \\ 'College of Pharmacy, Rady Faculty \\ of Health Sciences, University of \\ Manitoba, Winnipeg, MB, Canada; \\ ${ }^{2}$ Department of Community Health \\ Sciences, Rady Faculty of Health \\ Sciences, University of Manitoba, \\ Winnipeg, MB, Canada
}

Background: Pain is a main symptom of herpes zoster (HZ), and postherpetic neuralgia (PHN) is a frequent complication occurring in 5\% to $15 \%$ of cases, causing moderate to severe neuropathic pain. A population-based observational study was conducted to evaluate the treatment patterns and economic burden of prescription drug treatment of $\mathrm{HZ}$ and $\mathrm{PHN}$ pain in the province of Manitoba (Canada) over a period of 15 years.

Methods: Administrative health care data, including medical and hospital separation records, were examined to identify episodes of $\mathrm{HZ}$ using International Classification of Diseases-9/10 codes between April 1, 1997 and March 31, 2014. Episodes of PHN were identified using medical and prescription claims. Incident use of analgesic, antidepressant, or anticonvulsant drugs was used to determine prescription pain costs.

Results: The age-adjusted incidence of $\mathrm{HZ}$ increased from 4.7 episodes/ 1,000 person-years in $1997 / 98$ to $5.7 / 1,000$ person-years in $2013 / 14$. PHN occurred in $9.2 \%$ of $\mathrm{HZ}$ cases, a rate that did not change over the study period $(P=0.57)$. The annual cost to treat $\mathrm{HZ}$ pain rose by $174 \%$ from 1997/98, reaching CAD $\$ 332,981$ in 2011/12, 82.8\% (95\% confidence interval [CI] 81.2\%, $84.3 \%$ ) of which was related to PHN. The per episode cost of HZ rose by $111 \%$ from $\$ 31.59$ (95\% CI \$25.35, \$37.84) to \$66.81 (95\% CI \$56.84, \$76.78) and by $94 \%$ for PHN from $\$ 292$ (95\% CI $\$ 225, \$ 358)$ to $\$ 566$ (95\% CI \$478, \$655). These increases were driven by increasing use of anticonvulsants, primarily gabapentin, which accounted for $57 \%$ of the increase in cost. Conclusion: There has been an increase in the incidence of $\mathrm{HZ}$ and PHN and in the average cost associated with the prescription treatment of their resultant neuropathic pain. The primary driver of the increased episodic cost is the increased use of gabapentin. These changes have resulted in a substantial increase in the economic burden associated with $\mathrm{HZ}$ and PHN.

Keywords: shingles, cost, economics, neuropathic pain, gabapentin

\section{Introduction}

Herpes zoster (HZ) is an infectious disease caused by reactivation of latent varicella zoster infection. This common viral disease afflicts between $20 \%$ and $30 \%$ of the population at some point in their lifetime; up to $50 \%$ of individuals over 80 years of age are affected. ${ }^{1,2}$ The dermatological symptoms of $\mathrm{HZ}$ are typically preceded by prodromal symptoms that range from a tingling or burning sensation to a sharp stabbing pain. This is followed by a raised, reddish rash and vesicular lesions, typically over the region of skin affected by prodromal symptoms, 2 to 3 days later. This rash typically affects singular dermatomes resulting in a unilateral, stripe-like appearance that is diagnostic of HZ. Lesions continue to erupt for several days before the rash crusts over, generally within 7 to 10 days. Symptoms then begin to subside and total
Correspondence: Shawn Bugden College of Pharmacy, Rady Faculty of Health Sciences, University of Manitoba, 750 McDermot Avenue, Winnipeg, MB, R3E 0T5, Canada

Email shawn.bugden@umanitoba.ca 
resolution generally occurs within 3 to 4 weeks. ${ }^{3}$ While HZ is generally self-limiting, longer lasting complications can occur.

The most common complication of zoster is postherpetic neuralgia (PHN). PHN is defined as pain that persists after the resolution of the dermatological symptoms of $\mathrm{HZ}$. It is caused by damage to sensory neurons sustained during the initial active infection with pain persisting even after the virus has returned to its dormant state. ${ }^{4}$ There is no single standard definition of PHN. Pain persisting at 30 to 120 days measured from onset of $\mathrm{HZ}$ or from healing of rash is used in the literature..$^{5-7}$ Pain persisting at 90 days from initial $\mathrm{HZ}$ diagnosis is the most commonly used definition..$^{5-7}$

The incidence of $\mathrm{HZ}$ is estimated at 3.5 to 7 cases per 1,000 person-years (PY), with the incidence in females slightly higher than in males. ${ }^{8,9}$ The incidence of $\mathrm{HZ}$ increases steadily with age, rising to 6-7/1,000 PY by 60 years, and continuing to increase thereafter. ${ }^{2,10,11}$ The rate of conversion to PHN has been estimated at anywhere from $5 \%$ to $60 \%$ of HZ cases. ${ }^{7,11,12}$ This wide range is partially explained by the lack of standard diagnostic criteria. Studies that used pain persisting at 90 days to define $\mathrm{PHN}$ have a more conservative conversion rate with a narrower range of between $5 \%$ and $15 \%$. $^{7-9,11-13}$

$\mathrm{PHN}$ is a challenging condition to treat, and the effectiveness of pharmacological therapy is limited. The Canadian Pain Society consensus statement on the treatment of neuropathic pain recommends as first-line therapy for PHN one of the tricyclic antidepressants (TCAs), gabapentin, or pregabalin. ${ }^{14}$ Opioids and topical lidocaine are listed as second-line alternatives. The European Federation of Neurological Societies 2010 treatment guidelines for neuropathic pain largely echo these recommendations. Opioids, while effective, are not recommended as first-line therapy due to side effects, development of tolerance, and risk of dependency. ${ }^{15} \mathrm{~A}$ variety of other drugs are also discussed in the literature, including those used for other forms of neuropathy, although their efficacy for PHN is disputed. These include valproic acid/ divalproex sodium, duloxetine, venlafaxine, carbamazepine, tramadol, nonsteroidal anti-inflammatory drugs (NSAIDs), and selective serotonin reuptake inhibitors. ${ }^{6,14-18}$

While treatment of $\mathrm{PHN}$ remains difficult, it is possible to reduce the risk of $\mathrm{HZ}$, and with it $\mathrm{PHN}$, with the $\mathrm{HZ}$ vaccine (Zostavax II ${ }^{\circledR}$, Merck \& Co., Inc., Kenilworth, NJ, USA). This vaccine has been shown to reduce the relative risk of $\mathrm{HZ}$ by $51 \%$ and of PHN by almost $67 \%$. Vaccines may decrease the chance of developing $\mathrm{HZ}$, but $\mathrm{HZ}$ and the associated $\mathrm{PHN}$ are expected to continue to result in a significant burden to society in the near future.

To determine the incidence of $\mathrm{HZ}$ and rates of $\mathrm{PHN}$, a retrospective cohort study was conducted in Manitoba, Canada, over a period of 17 years. The objectives were to determine the burden of disease of zoster-related pain and to understand the major cost drivers in the changing cost of treating HZ.

\section{Methods}

A population-based cohort study was conducted in Manitoba (Canada) using administrative health care data from Manitoba's universal public health care system gathered from April 1, 1995 to March 31, 2014 in the course of providing routine medical care. These data were accessed via the Manitoba Centre for Health Policy Population Health Research Data Repository, a collection of databases containing records of contacts of Manitoba residents with the health care system. ${ }^{19}$ Databases utilized included the Drug Program Information Network, which processes all community pharmacy-based prescriptions for insurance coverage and drug utilization review; the Medical Services database which contains records of all fee-for-service medical provider claims; hospital discharge abstracts containing separations data for all hospitalizations; and the Manitoba Health Registry which contains basic demographic information on all persons registered with Manitoba Health, Healthy Living and Seniors. All records are de-identified but contain a unique scrambled personal health number allowing for the cross-linkage of records over time and across data sets.

Episodes of zoster were identified using diagnostic codes in medical claims and hospital separations. Individuals with one or more International Classification of Diseases-9 (ICD9) codes starting with 053 or ICD-10-Clinical Modification (ICD-10-CM) codes starting with B02 were considered as $\mathrm{HZ}$ cases, the date of the first being the episode start date. The use of ICD diagnostic codes to diagnose zoster was both highly selective (positive predictive value 93\%) and sensitive (97.5\%). ${ }^{20}$ Multiple episodes per individual were allowed if 2 years had elapsed between episodes. To ensure only incident episodes were included in the analysis, a 2-year washout period was used; thus, any episodes identified prior to $1997 / 98$ were excluded. Episodes where individuals were under 20 years at diagnosis were excluded to avoid misclassification of varicella zoster cases as HZ. Cases of PHN were identified by $\mathrm{HZ}$ pain drug treatment or medical claims with HZ ICD codes appearing past 90 days from diagnosis. Ongoing treatment is defined in the following section. 
Drug treatment was categorized using Anatomical Therapeutic Chemical classes. Prescriptions for opioids (Anatomical Therapeutic Chemical codes starting with N02A), NSAIDs (M01), anticonvulsants (N03A), antidepressants (N06A), nabilone (A04AD), local anesthetics (D04A), glucocorticoids (H02AB), and acetylsalicylic acid and acetaminophen (N02B) associated with HZ episodes identified in the previous step were collected. Those dispensed between 90 days pre-HZ diagnoses and 2 years postdiagnosis were evaluated against several criteria to assess if they were related to HZ-PHN or were only coincident.

The use of each drug class was categorized as either incident or prevalent for that episode by evaluating prescriptions in the 90 days preceding diagnosis. A drug class was categorized as prevalent if a person received a total of 30 days' supply or more within those 90 days, and all withinclass prescriptions were excluded from the analysis for that episode. From the remaining incident class, all prescriptions from diagnosis to 1) 90 days postdiagnosis or 2) date of last $\mathrm{HZ}$ medical claim were classified as HZ-PHN treatment. Prescriptions with dispensation dates outside this window had to meet the continuous use criteria. A grace period of $100 \%$ of the prescription duration was added to its duration to create an end date. To be considered a continuous treatment, there should be no gaps between the end date of previous prescriptions and receipt of the next. Discontinuity signaled the end of drug treatment and of that episode; all later prescriptions were removed from further analysis of this episode.

Data were summarized by Manitoba Health fiscal years, which run from April 1 to March 31 for each year. Results are reported as occurring within the fiscal year of diagnosis. Incidence rates for $\mathrm{HZ}$ were calculated for 1997/98 through to 2013/14, and age adjusted using the population of Manitoba in 1997 as our standard. As 2 years follow-up was required to capture all data related to episodes of $\mathrm{HZ}$ with PHN, only episodes diagnosed from 1997/98 to 2011/12 contributed to the PHN analysis. Regression analysis was conducted on rates of conversion from $\mathrm{HZ}$ to $\mathrm{PHN}$ across this interval.

Drug costs were calculated directly from prescription records, included cost of both drug and dispensing fee, and were adjusted to 2013 Canadian dollars using Statistics Canada's consumer price index for prescription drug costs in Manitoba. Annual total prescription costs were tabulated for all $\mathrm{HZ}$ episodes, as well as within $\mathrm{HZ}$ episodes that converted to PHN (HZ-PHN). Prescription costs per episode were calculated, and $t$-tests were conducted to determine the significance of differences between mean costs in 1997/98 and 2011/12. Regression on the proportion of total cost due to HZ-PHN was used to look for trends over time. The relationship between age at diagnosis and mean treatment cost was examined by regression analysis.

Prescription data were stratified by drug class for HZPHN episodes, looking at anticonvulsants, antidepressants, NSAIDs, and opioids. The number of treated episodes was determined for each year. Regressions on the number of classwise treated episodes were used to examine trends over time.

SAS version 9.4 (SAS Institute Inc., Cary, NC, USA) was used for all data analysis. Approvals were granted by the University of Manitoba Health Research Ethics Board and the Manitoba Health Information Privacy Committee. Individual written consent from patients was not required by the ethics board or the privacy committee for this retrospective analysis using de-identified data.

\section{Results}

Between April 1, 1997 and March 31, 2014, there were 73,893 episodes of $\mathrm{HZ}$ diagnosed in Manitoba resulting in a mean of 4,347 (95\% confidence interval [CI] 4,067, 4,626) episodes per year. There were 5,749 episodes of HZ diagnosed in 2013/14, a 50\% increase from the 3,844 episodes diagnosed in 1997/98. The age-standardized incidence rate of $\mathrm{HZ}$ increased by $21 \%$, from 4.70 episodes $/ 1,000 \mathrm{PY}$ in 1997/98 to 5.70 episodes/1,000 PY in 2013/14.

Total prescription costs for treating pain resulting from $\mathrm{HZ}$ and PHN are shown in Table 1. The annual cost of prescription drugs used to treat all HZ-related pain rose by over $174 \%$, from CAD \$121,438 in 1997/98 to $\$ 332,981$ in 2011/12. The drug cost of HZ-PHN episodes rose by $191 \%$, from $\$ 96,554$ to $\$ 281,342$. PHN accounted for a mean of $82.8 \%$ (95\% CI $81.2 \%$, $84.3 \%$ ) of drug costs. This percentage did not change significantly over the course of the study period $\left(P=0.57, R^{2}=0.026\right)$.

From 1997/98 through to 2011/12, the period for which full PHN results could be obtained, there were 6,038 episodes of $\mathrm{HZ}$ that met the study criteria for PHN, corresponding to a conversion rate of $9.6 \%$. A linear regression analysis on conversion rates revealed no significant trend over this period of time ( $P=0.45, R^{2}=0.046$ ). However, the increase in the total number of $\mathrm{HZ}$ episodes resulted in a greater number of PHN cases over time, rising from 331 in 1997/98 to 497 in 2011/12.

The mean cost of treating HZ-related pain increases linearly with age at diagnosis, each additional year increasing the mean episode cost by $\$ 1.04\left(P<0.0001, R^{2}=0.65\right)$ (Figure $1)$. The mean cost for persons aged 20 to 29 years was $\$ 24.15$ (95\% CI \$19.95, \$28.34), while for persons aged 60 to 69 years the cost was $143 \%$ higher at $\$ 58.72$ (95\% CI \$52.92, \$64.53).

The average cost of treating an episode of HZ, and separately those episodes with PHN, increased over the study 
Table I Total cost and cost per episode of treating herpes zoster-related pain by year

\begin{tabular}{|c|c|c|c|c|c|c|c|c|}
\hline \multirow[b]{2}{*}{ Year } & \multicolumn{4}{|c|}{ Herpes zoster episodes } & \multicolumn{4}{|c|}{ Herpes zoster with PHN } \\
\hline & Episodes & Rxs & Total cost & Mean cost $(95 \% \mathrm{Cl})$ & Episodes & Rxs & Total cost & Mean PHN cost $(95 \% \mathrm{Cl})$ \\
\hline $1997 / 1998$ & 3,844 & 6,082 & $\$ 121,438$ & $\$ 31.59(\$ 25.35, \$ 37.84)$ & 331 & 3,925 & $\$ 96,555$ & $\$ 291.71(\$ 225.45, \$ 357.96)$ \\
\hline $1998 / 1999$ & 3,781 & 6,309 & $\$ 130,276$ & $\$ 34.46(\$ 28.38, \$ 40.53)$ & 349 & 4,229 & $\$ 107,007$ & $\$ 306.61(\$ 248.06, \$ 365.16)$ \\
\hline $1999 / 2000$ & $3,94 I$ & 7,820 & $\$ 245,329$ & $\$ 62.25(\$ 50.74, \$ 73.76)$ & 367 & 5,526 & $\$ 215,149$ & $\$ 586.24(\$ 475.95, \$ 696.53)$ \\
\hline $2000 / 2001$ & 3,954 & 7,629 & $\$ 224,626$ & $\$ 56.81(\$ 46.70, \$ 66.92)$ & 399 & 5,440 & $\$ 192,830$ & $\$ 483.28(\$ 393.34, \$ 573.23)$ \\
\hline $2001 / 2002$ & 4,060 & 8,282 & $\$ 271,792$ & $\$ 66.94(\$ 52.67, \$ 81.22)$ & 429 & 6,064 & $\$ 236,570$ & $\$ 551.45(\$ 425.20, \$ 677.69)$ \\
\hline $2002 / 2003$ & 4,126 & 8,015 & $\$ 254,967$ & $\$ 61.80(\$ 52.07, \$ 7 \mid .52)$ & 410 & 5,554 & $\$ 215,795$ & $\$ 526.33(\$ 440.91, \$ 611.75)$ \\
\hline $2003 / 2004$ & 4,070 & 7,783 & $\$ 23 I, 833$ & $\$ 56.96(\$ 47.57, \$ 66.35)$ & 375 & 5,541 & $\$ 191,562$ & $\$ 510.83(\$ 421.55, \$ 600.12)$ \\
\hline $2004 / 2005$ & 4,087 & 7,070 & $\$ 194,372$ & $\$ 47.56(\$ 40.78, \$ 54.34)$ & 379 & 4,670 & $\$ 153,89 \mid$ & $\$ 406.05(\$ 343.97, \$ 468.12)$ \\
\hline $2005 / 2006$ & $4,|3|$ & 7,886 & $\$ 214,436$ & $\$ 5 I .91(\$ 44.30, \$ 59.51)$ & 432 & 5,581 & $\$ 172,052$ & $\$ 398.27(\$ 334.80, \$ 461.74)$ \\
\hline $2006 / 2007$ & 4,143 & 7,918 & $\$ 207,393$ & $\$ 50.06(\$ 42.52, \$ 57.60)$ & 377 & 5,586 & $\$ 165,257$ & $\$ 438.35(\$ 366.84, \$ 509.86)$ \\
\hline $2007 / 2008$ & 4,207 & 7,968 & $\$ 193,942$ & $\$ 46.10(\$ 39.89, \$ 52.31)$ & 377 & 5,674 & $\$ 152,928$ & $\$ 405.64(\$ 348.23, \$ 463.06)$ \\
\hline $2008 / 2009$ & 4,295 & 9,435 & $\$ 252,994$ & $\$ 58.90(\$ 50.77, \$ 67.04)$ & 440 & 7,147 & $\$ 209,013$ & $\$ 475.03(\$ 407.32, \$ 542.74)$ \\
\hline $2009 / 2010$ & 4,584 & 9,393 & $\$ 267,865$ & $\$ 58.43(\$ 49.20, \$ 67.67)$ & 427 & 7,024 & $\$ 222,968$ & $\$ 522.17(\$ 434.64, \$ 609.7 \mathrm{I})$ \\
\hline $2010 / 2011$ & 4,622 & 9,102 & $\$ 276,383$ & $\$ 59.80(\$ 49.42, \$ 70.17)$ & 449 & 6,617 & $\$ 230,827$ & $\$ 514.09(\$ 416.88, \$ 611.30)$ \\
\hline $2011 / 2012$ & 4,984 & 11,065 & $\$ 332,981$ & $\$ 66.8 \mathrm{I}(\$ 56.84, \$ 76.78)$ & 497 & 8,398 & $\$ 281,342$ & $\$ 566.08(\$ 477.5 \mathrm{I}, \$ 654.66)$ \\
\hline
\end{tabular}

Notes: Costs include all prescriptions from date of diagnosis to end of episode and include prescriptions for postherpetic neuralgia when present. The herpes zoster with PHN group includes only those cases of herpes zoster that converted to neuralgia and includes prescriptions from the start of the herpes zoster episode. Costs are reported in 2013 Canadian dollars.

Abbreviations: $\mathrm{PHN}$, postherpetic neuralgia; Rxs, prescriptions; $\mathrm{Cl}$, confidence interval.

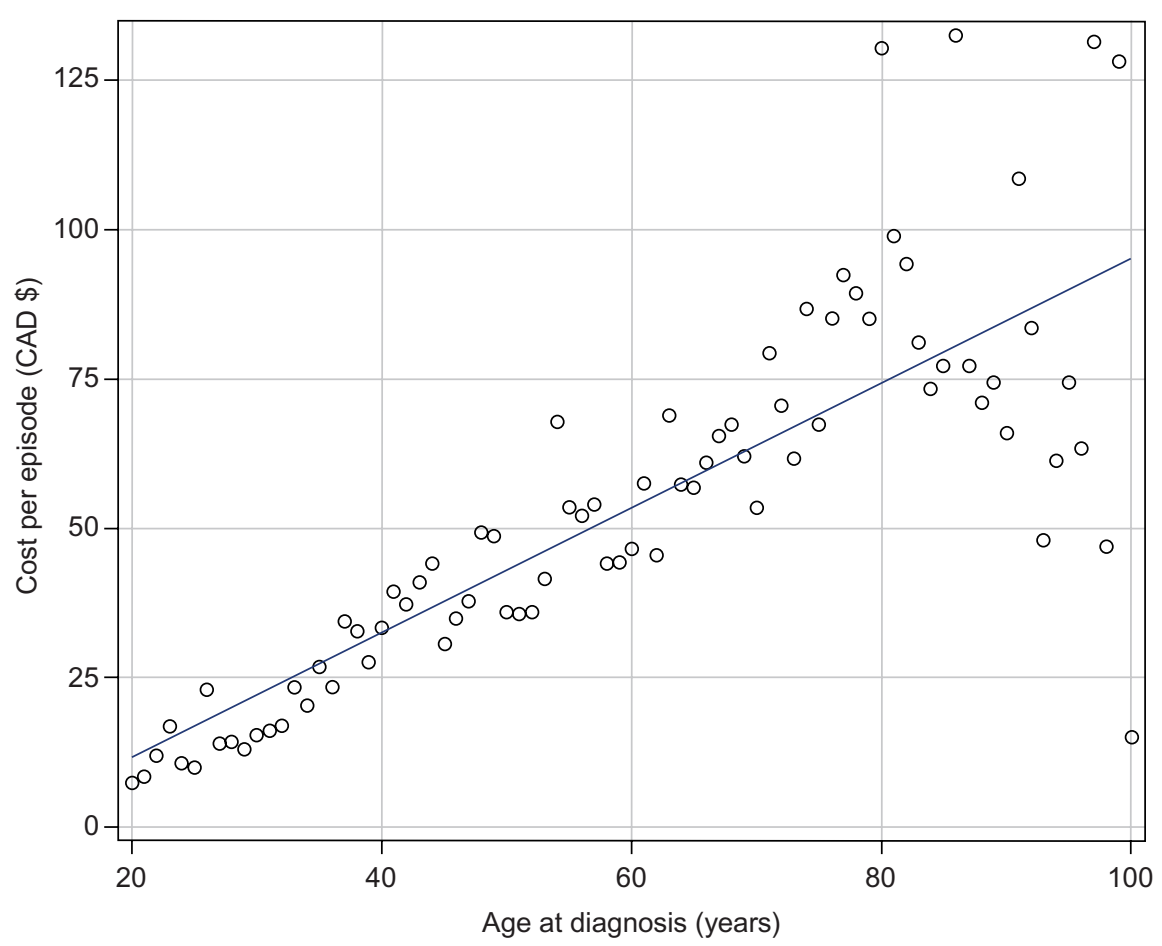

Figure I Mean cost per episode of herpes zoster-associated pain treatment by age at diagnosis. Costs adjusted for inflation to 2013 Canadian dollars.

period (Table 1). The mean cost per episodes to treat all HZrelated pain rose significantly by $111 \%(t=5.369, P<0.0001)$ from $1997 / 98$ to $2011 / 12$, and by $94 \%$ for HZ-PHN episodes $(t=4.36, P<0.0001)$.

Changes in the treatment of HZ-PHN over time were examined by linear regression (Figure 2). Only anticonvulsant use changed significantly with a $212 \%$ increase in the number of anticonvulsant-treated episodes from 1997/98 to 2011/12 $\left(P<0.0001, R^{2}=0.92\right)$.

The duration of treatment for HZ-PHN varied widely between drug classes (Figure 3). The median days supplied was highest for antidepressants (178 days) and anticonvulsants (134 days), with shorter durations for NSAIDs (50 days) and opioids (28 days). The median duration of anticonvulsant 


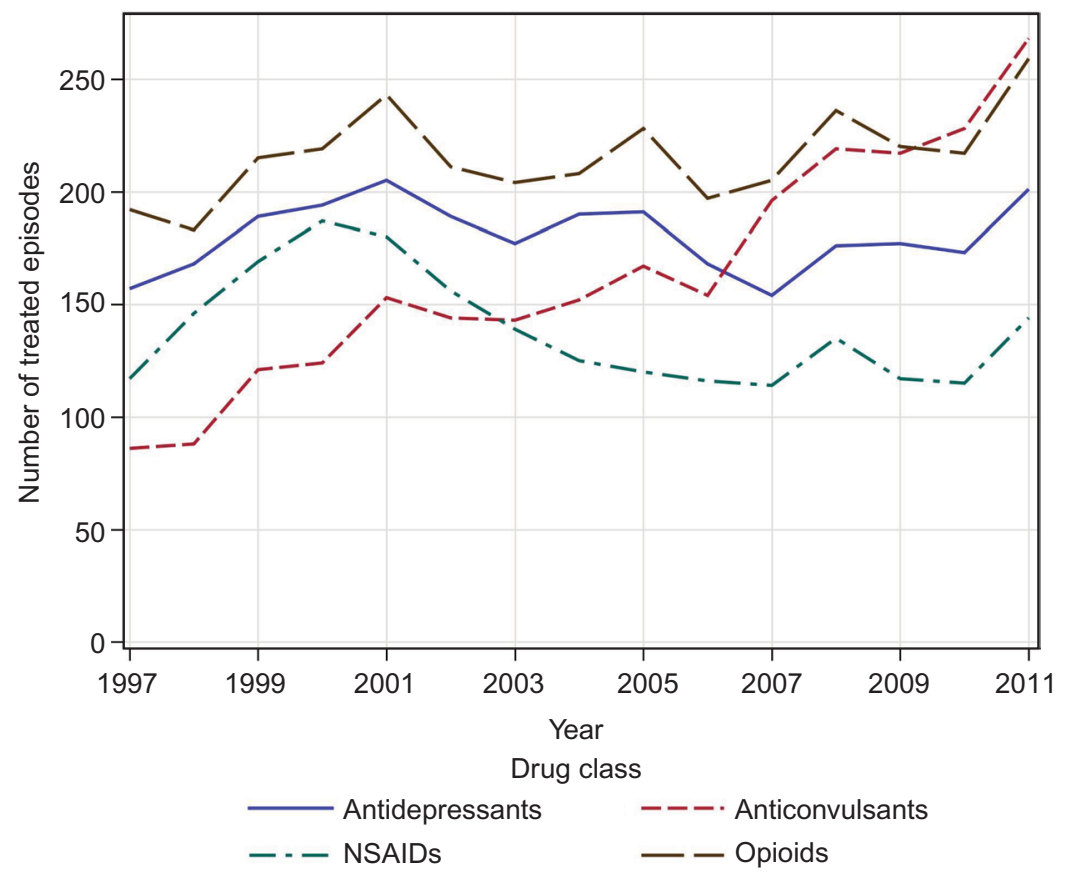

Figure 2 Treatment of postherpetic neuralgia episodes by drug class.

Notes: Numbers of episodes treated by drug class. A single episode can be treated with more than one class of drug, thus a single episode can contribute data to more than one category.

Abbreviation: NSAID, nonsteroidal anti-inflammatory drug.

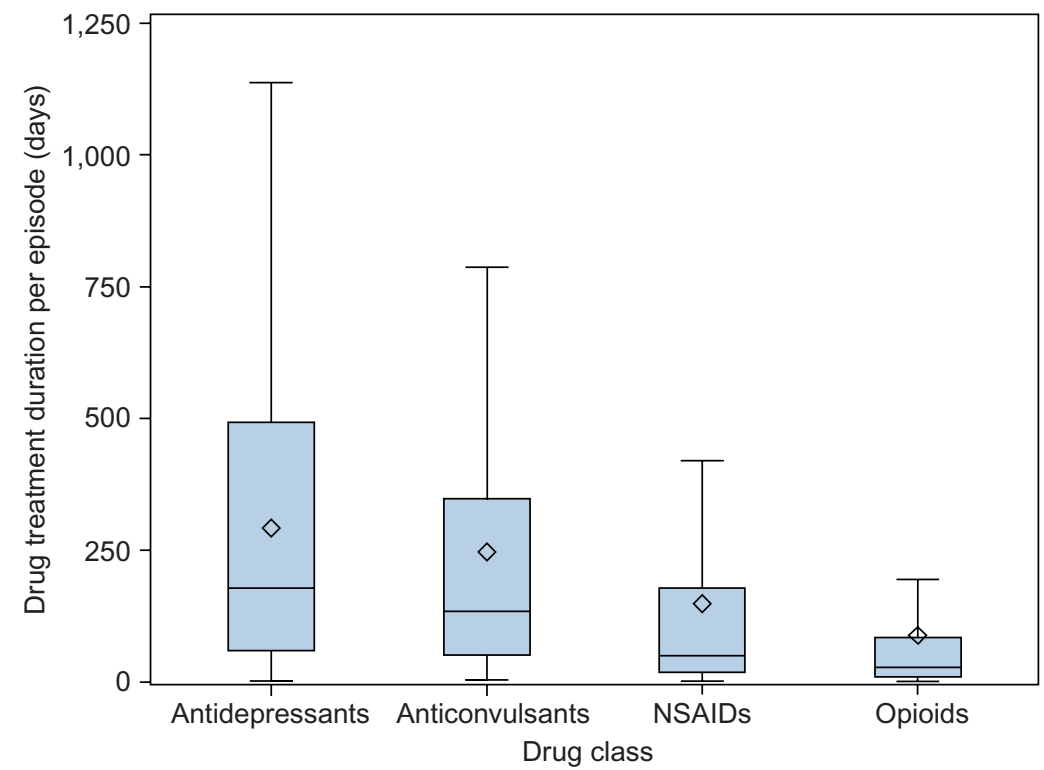

Figure 3 Distribution of days of drug treatment per episode by class.

Notes: Boxplot of the number of days of drug treatment per episode by drug class for herpes zoster with postherpetic neuralgia. $\diamond=$ mean.

Abbreviation: NSAID, nonsteroidal anti-inflammatory drug.

treatment increased from 55 to 141 days between 1997/98 and 2011/12. There was much less change observed in the median treatment duration of other drug classes, with antidepressants increasing from 161 to 190 days, opioids remaining relatively stable with an increase from 26.5 to 27 days, and NSAIDs decreasing from 38.5 to 30 days.
The overall annual cost of opioid, NSAID, and antidepressant prescriptions underwent a moderate increase with antidepressants increasing by $91 \%$, opioids by $67 \%$, and NSAIDs by $35 \%$ (Table 2). However, these changes were small compared to the increase in anticonvulsant use, which underwent a $755 \%$ rise in class cost, increasing from 
$\$ 15,520$ to $\$ 132,685$. This increase accounts for $56 \%$ of the total increase in prescription spending for the treatment of all HZ-associated pain.

The most frequently prescribed anticonvulsant drug for HZ-PHN also changed over time. In 1997, carbamazepine prescriptions accounted for 62\% (260 prescriptions) of all anticonvulsant prescriptions, while gabapentin accounted for only $21 \%$ (89 prescriptions). In contrast, in 2011 , only $3.2 \%$ (80 prescriptions) of all anticonvulsant prescriptions were for carbamazepine. Gabapentin accounted for $73 \%(1,798)$ of prescriptions, while pregabalin, which was not available in 1997 , accounted for $9.2 \%$ (226).

\section{Discussion}

The economic burden of treating HZ-associated pain, and PHN in particular, increased significantly from 1997/98 to 2011/12 (Table 1). This increase is the combined result of increases in both the incidence of diagnosed $\mathrm{HZ}$ and in the mean episode cost of prescription drug treatment of pain. A dramatic increase in gabapentin was found to be a primary driver of mean episode cost.

Over the 17-year period between 1997/98 and 2013/14, there has been a sustained increase in the incidence of $\mathrm{HZ}$, with a resulting increase in the number of PHN cases. Changes in size and increasing age $\mathrm{e}^{21}$ of the provincial population explain the majority of this increase. However, after taking both these factors into account, a significant increase of $21 \%$ in the age-adjusted incidence per thousand people was observed. As the rate of conversion from $\mathrm{HZ}$ to $\mathrm{PHN}$ did not change over the study period, the increased number of $\mathrm{HZ}$ episodes resulted in an increase in the number of PHN cases. This increase in PHN cases is an important factor in determining the economic burden of $\mathrm{HZ}$, as these cases were responsible for over $80 \%$ of $\mathrm{HZ}$-related analgesic prescription costs.

Not only were there more HZ and PHN cases, there was also a significant increase in the mean cost per episode related to the treatment of pain. This higher episode cost was driven by the increased use of anticonvulsant drugs, primarily gabapentin. While the number of users of anticonvulsants rose, the use of other classes of drugs remained relatively unchanged (Table 2). Not only did the number of persons using anticonvulsant drugs increased, the median duration of treatment increased with them as well.

From an evidence-based perspective, this change might seem surprising. There is considerable debate on whether this increased expenditure is justified. There is limited evidence demonstrating superior efficacy and involves much greater cost compared to the other first-line therapies such as TCAs. ${ }^{22}$ In fact, one systematic review estimated the number needed to treat (NNT) for a $50 \%$ reduction of pain for gabapentin to be 4.39 (95\% CI 3.34, 6.07), while that for TCAs was 2.64 (95\% CI $2.1,3.54) .{ }^{23}$ Gabapentin was introduced to the Canadian market in December 1994 when it was approved for the adjunctive treatment of partial seizures. ${ }^{24}$ It was aggressively marketed to physicians for a number of nonapproved indications, including PHN, by Parke Davis. This promotion eventually led to a US federal lawsuit against the company for off-label marketing in 1996. ${ }^{25}$ It was not until 2004 that the US Food and Drug Administration (FDA) approved gabapentin for $\mathrm{PHN},{ }^{25}$ although it has never received this indication in Canada. Some of this promo-

Table 2 Treatment of herpes zoster-related pain by drug class

\begin{tabular}{|c|c|c|c|c|c|c|c|c|c|c|}
\hline \multirow[b]{2}{*}{ Year } & \multicolumn{2}{|c|}{ Antidepressants } & \multicolumn{2}{|c|}{ Anticonvulsants } & \multicolumn{2}{|c|}{ NSAIDs } & \multicolumn{2}{|c|}{ Opioids } & \multicolumn{2}{|c|}{ Miscellaneous } \\
\hline & Users & Cost (CAD) & Users & Cost (CAD) & Users & Cost (CAD) & Users & Cost (CAD) & Users & Cost (CAD) \\
\hline 1997 & 348 & $\$ 35,607$ & 153 & $\$ 15,520$ & 400 & $\$ 23,122$ & 866 & $\$ 33,208$ & 544 & $\$ 13,980$ \\
\hline 1998 & 378 & $\$ 29,458$ & 168 & $\$ 29,124$ & 380 & $\$ 34,326$ & 838 & $\$ 23,896$ & 520 & $\$ 13,47 \mid$ \\
\hline 1999 & 404 & $\$ 39,612$ & 235 & $\$ 83,398$ & 475 & $\$ 64,916$ & 893 & $\$ 43,752$ & 571 & $\$ 13,65 \mid$ \\
\hline 2000 & 424 & $\$ 46,315$ & 250 & $\$ 64,642$ & 479 & $\$ 66,661$ & 893 & $\$ 34,441$ & 505 & $\$ 12,567$ \\
\hline 2001 & 440 & $\$ 48,663$ & 293 & $\$ 85,722$ & 469 & $\$ 47,112$ & 885 & $\$ 70,139$ & 544 & $\$ 20,157$ \\
\hline 2002 & 435 & $\$ 52,683$ & 298 & $\$ 82,635$ & 453 & $\$ 48,795$ & 920 & $\$ 56,048$ & 574 & $\$ 14,806$ \\
\hline 2003 & 425 & $\$ 46,661$ & 303 & $\$ 71,625$ & 398 & $\$ 32,101$ & 819 & $\$ 62,947$ & 532 & $\$ 18,498$ \\
\hline 2004 & 444 & $\$ 49,589$ & 326 & $\$ 63,383$ & 368 & $\$ 28,335$ & 889 & $\$ 36,793$ & 552 & $\$ 16,273$ \\
\hline 2005 & 445 & $\$ 44,140$ & 364 & $\$ 78,247$ & 377 & $\$ 29,826$ & 902 & $\$ 40,096$ & 540 & $\$ 22,127$ \\
\hline 2006 & 419 & $\$ 49,873$ & 389 & $\$ 70,737$ & 364 & $\$ 27,909$ & 848 & $\$ 31,437$ & 502 & $\$ 27,437$ \\
\hline 2007 & 396 & $\$ 37,136$ & 485 & $\$ 86,579$ & 325 & $\$ 24,099$ & 829 & $\$ 31,008$ & 501 & $\$ 15,119$ \\
\hline 2008 & 397 & $\$ 5 I, 837$ & 533 & $\$ 1 \mid 2,910$ & 389 & $\$ 27,118$ & 828 & $\$ 35,141$ & 515 & $\$ 25,988$ \\
\hline 2009 & 430 & $\$ 54,128$ & 540 & $\$ 122,855$ & 348 & $\$ 24,299$ & 866 & $\$ 41,026$ & 518 & $\$ 25,558$ \\
\hline 2010 & 428 & $\$ 49,949$ & 581 & $\$ 123,577$ & 326 & $\$ 23,549$ & 853 & $\$ 42,545$ & 526 & $\$ 36,763$ \\
\hline 2011 & 447 & $\$ 67,903$ & 694 & $\$ 132,685$ & 383 & $\$ 31,252$ & 926 & $\$ 55,318$ & 582 & $\$ 45,824$ \\
\hline
\end{tabular}

Notes: Total treated herpes zoster episodes (users) and drug class cost by year. Numbers are for all herpes zoster-related pain, including postherpetic neuralgia. A single episode can be treated with more than one class of drug, possibly contributing data to multiple categories. Costs adjusted for inflation to 2013 Canadian dollars. Abbreviation: NSAID, nonsteroidal anti-inflammatory drug. 
tion suggested that TCA use for PHN was inappropriate in older patients from a safety perspective. ${ }^{22}$ However, gabapentin is not without its own adverse effects, and a systematic review suggests the number needed to harm $(\mathrm{NNH})$ for major harms is actually similar for gabapentin $(\mathrm{NNH}=12.25,95 \% \mathrm{CI} 7.69$, $30.2)$ and TCAs $(\mathrm{NNH}=16.9,95 \% \mathrm{CI} 8.85,178) .{ }^{23}$

Concerns have been raised about the use of opioids in noncancer pain. ${ }^{26}$ These include the risk of dependency, the development of tolerance, and the potential for abuse, and are part of the rationale for listing opioids as second-line PHN treatment options. ${ }^{15,27}$ However, opioids were used frequently to treat HZ-PHN episodes (Figure 2). While the high rate of use may be concerning, the median of duration of opioid treatment (Figure 3) reveals generally short-term use, possibly reflecting appropriate opioid stewardship.

\section{Limitations}

This study has a number of strengths and limitations. Population level data from a universal single-payer health care system ensures virtually all contacts of residents with this health care system, and all prescription dispensations are captured in the study. These data sets also allow us to directly measure health system costs rather than inferring results from survey data or sampling, as it has been done in many previous studies. ${ }^{12,28-30}$ There are also several limitations to this study. We were unable to measure the true incidence of $\mathrm{HZ}$ in the population as only persons who sought medical treatment appeared in the administrative data. However, previous studies have reported that up to $95 \%-99 \%$ of persons with $\mathrm{HZ}$ will seek medical attention. ${ }^{8,28}$ Second, HZ diagnostic codes or prescription treatment past 90 days postdiagnosis were used as a proxy for a PHN diagnosis as we only had access to the first three digits of ICD codes in our data. Thus, rates reported are of HZ cases requiring ongoing medical management, sometimes referred to as clinically significant PHN. This methodology is common in administrative data studies looking at PHN. ${ }^{8}$ Finally, the use of nonprescription medications, including low-dose ibuprofen and naproxen, acetaminophen, acetylsalicylic acid, and topical treatments, is not captured. However, these costs are outside the scope of this study.

\section{Conclusion}

The total costs of treating pain associated with $\mathrm{HZ}$ and $\mathrm{PHN}$ rose by $174 \%$ between $1997 / 98$ and $2011 / 12$. There are two primary components to this increasing economic burden. The first is the increasing number of $\mathrm{HZ}$ cases. While some of this can be attributed to an aging population, the agestandardized incidence of $\mathrm{HZ}$ has increased. The second component is the increasing average cost of treating an episode of HZ-PHN driven by the use of higher cost anticonvulsants for longer periods of time. Management of the economic burden of HZ-PHN analgesic treatment should include the use of evidence-based cost-effective therapies and measures such as vaccination that can help reduce the incidence of $\mathrm{HZ}$.

\section{Acknowledgments}

The authors acknowledge the Manitoba Centre for Health Policy for use of data contained in the Population Health Research Data Repository under project \#H2014:411 (Health Information Privacy Committee \# 2014/2015-35). The results and conclusions are those of the authors and no official endorsement by the Manitoba Centre for Health Policy, Manitoba Health, Healthy Living and Seniors, or other data providers is intended or should be inferred. Data used in this study are from the Population Health Research Data Repository housed at the Manitoba Centre for Health Policy, University of Manitoba, and were derived from data provided by Manitoba Health. The authors would also like to thank Heather Prior from the Manitoba Centre for Health Policy for her assistance and preliminary data extraction.

\section{Disclosure}

This work was supported by a investigator-initiated grant grant from Merck Canada. The authors report no other conflicts of interest in this work.

\section{References}

1. Brisson M, Edmunds WJ, Law B, et al. Epidemiology of varicella zoster virus infection in Canada and the United Kingdom. Epidemiol Infect. 2001;127(02):305-314.

2. Johnson RW, Alvarez-Pasquin M-J, Bijl M, et al. Herpes zoster epidemiology, management, and disease and economic burden in Europe: a multidisciplinary perspective. Ther Adv Vaccines. 2015;3(4):109-120.

3. Dworkin RH, Johnson RW, Breuer J, et al. Recommendations for the management of herpes zoster. Clin Infect Dis. 2007;44(Suppl 1): S1-S26.

4. Bennett G, Watson C. Herpes zoster and postherpetic neuralgia: past, present and future. Pain Res Manag. 2009;14(4):275-282. Available from: http://www.ncbi.nlm.nih.gov/pmc/articles/pmc2734513/. Accessed November 18, 2015.

5. Snedecor SJ, Sudharshan L, Cappelleri JC, et al. Systematic review and meta-analysis of pharmacological therapies for pain associated with postherpetic neuralgia and less common neuropathic conditions. Int $J$ Clin Pract. 2014;68(7):900-918.

6. Tontodonati M, Ursini T, Polilli E, et al. Post-herpetic neuralgia. Int $J$ Gen Med. 2012;5:861-871.

7. Gauthier A, Breuer J, Carrington D, Martin M, Rémy V. Epidemiology and cost of herpes zoster and post-herpetic neuralgia in the United Kingdom. Epidemiol Infect. 2009;137(1):38-47.

8. Ultsch B, Köster I, Reinhold T, et al. Epidemiology and cost of herpes zoster and postherpetic neuralgia in Germany. Eur J Health Econ. 2013;14(6):1015-1026. 
9. Weitzman D, Shavit O, Stein M, Cohen R, Chodick G, Shalev V. A population based study of the epidemiology of herpes zoster and its complications. J Infect. 2013;67(5):463-469.

10. Tanuseputro P, Zagorski B, Chan KJ, Kwong JC. Population-based incidence of herpes zoster after introduction of a publicly funded varicella vaccination program. Vaccine. 2011;29(47):8580-8584.

11. Edmunds WJ, Brisson M, Rose JD. The epidemiology of herpes zoster and potential cost-effectiveness of vaccination in England and Wales. Vaccine. 2001;19(23-24):3076-3090.

12. Cebrián-Cuenca AM, Díez-Domingo J, San-Martín-Rodríguez M, PuigBarberá J, Navarro-Pérez J. Epidemiology and cost of herpes zoster and postherpetic neuralgia among patients treated in primary care centres in the Valencian community of Spain. BMC Infect Dis. 2011;11(1):302.

13. Gialloreti LE, Merito M, Pezzotti P, et al. Epidemiology and economic burden of herpes zoster and post-herpetic neuralgia in Italy: a retrospective, population-based study. BMC Infect Dis. 2010;10:230.

14. Moulin D, Boulanger A, Clark AJ, et al. Pharmacological management of chronic neuropathic pain: revised consensus statement from the Canadian Pain Society. Pain Res Manag. 2014;19(6):328-335.

15. Attal N, Cruccu G, Baron R, et al. EFNS guidelines on the pharmacological treatment of neuropathic pain: 2010 revision. Eur J Neurol. 2010; 17(9):1113-e88.

16. Boivin G, Jovey R, Elliott CT, Patrick DM. Management and prevention of herpes zoster: a Canadian perspective. Can J Infect Dis Med Microbiol. 2010;21(1):45-52.

17. Philip A, Thakur R. Post herpetic neuralgia. J Palliat Med. 2011;14(6): 765-773.

18. Massengill JS, Kittredge JL. Practical considerations in the pharmacological treatment of postherpetic neuralgia for the primary care provider. J Pain Res. 2014;7:125-132.

19. Roos LL, Brownell M, Lix L, Roos NP, Walld R, Macwilliam L. From health research to social research: privacy, methods, approaches. Soc Sci Med. 2008;66:117-129.
20. Klompas M, Kulldorff M, Vilk Y, Bialek SR, Harpaz R. Herpes zoster and postherpetic neuralgia surveillance using structured electronic data. Mayo Clin Proc. 2011;86(12):1146-1153.

21. Statistics Canada. Annual Demographic Estimates: Canada, Provinces and Territories. Ottawa, ON: Statistics Canada; 2015.

22. O'Connor AB, Noyes K, Holloway RG. A cost-effectiveness comparison of desipramine, gabapentin, and pregabalin for treating postherpetic neuralgia. JAm Geriatr Soc. 2007;55(8):1176-1184.

23. Hempenstall K, Nurmikko TJ, Johnson RW, A'Hern RP, Rice ASC. Analgesic therapy in postherpetic neuralgia: a quantitative systematic review. PLoS Med. 2005;2(7):0628-0644.

24. Drug Products Database. Heal Canada Website. Available from: http:// webprod5.hc-sc.gc.ca/dpd-bdpp/index-eng.jsp. Accessed November 2, 2015.

25. Torborg DS. The dark side of the boom: the peculiar dilemma of modern False Claims Act litigation. J Law Health. 2013;26:181-218.

26. National Opioid Use Guideline Group. Canadian Guideline for Safe and Effective Use of Opioids for Chronic Non-Cancer Pain-Part A Executive Summary. 2010:1-38. Available from: http://nationalpaincentre. mcmaster.ca/documents/opioid_guideline_part_a_v4_5.pdf. Accessed April 25, 2016.

27. Moulin DE, Clark AJ, Gilron I, et al. Pharmacological management of chronic neuropathic pain - consensus statement and guidelines from the Canadian Pain Society. Pain Res Manag. 2007;12(1):13-21.

28. Bilcke J, Ogunjimi B, Marais C, et al. The health and economic burden of chickenpox and herpes zoster in Belgium. Epidemiol Infect. 2012;140(11):2096-2109.

29. Di Legami V, Gianino MM, Atti MCD, et al. Epidemiology and costs of herpes zoster: background data to estimate the impact of vaccination. Vaccine. 2007;25(43):7598-7604.

30. Lionis CD, Vardavas CI, Symvoulakis EK, et al. Measuring the burden of herpes zoster and post herpetic neuralgia within primary care in rural Crete, Greece. BMC Fam Pract. 2011;12(1):136.
Journal of Pain Research

\section{Publish your work in this journal}

The Journal of Pain Research is an international, peer reviewed, open access, online journal that welcomes laboratory and clinical findings in the fields of pain research and the prevention and management of pain. Original research, reviews, symposium reports, hypothesis formation and commentaries are all considered for publication.

\section{Dovepress}

The manuscript management system is completely online and includes a very quick and fair peer-review system, which is all easy to use. Visit http://www.dovepress.com/testimonials.php to read real quotes from published authors. 